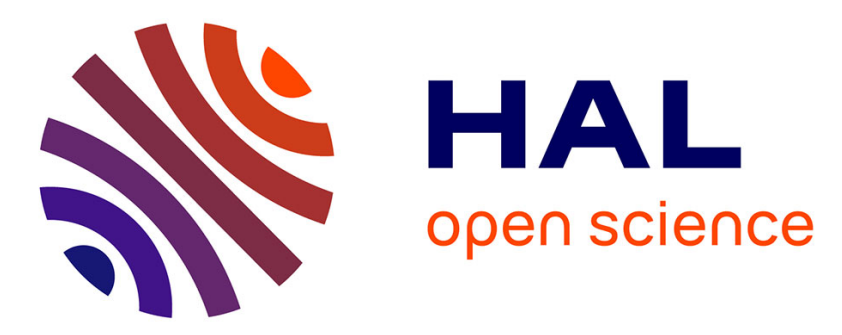

\title{
Advanced neutron diffraction techniques for strain measurements in polycrystalline materials
}

\author{
P. Mikula, P. Lukás, M. Vrána, P. Klimanek, T. Kschidock, K. Macek, J.
}

Janovec, J. Osborn, G. Swallowe

\section{- To cite this version:}

P. Mikula, P. Lukás, M. Vrána, P. Klimanek, T. Kschidock, et al.. Advanced neutron diffraction techniques for strain measurements in polycrystalline materials. Journal de Physique IV Proceedings, 1993, 03 (C7), pp.C7-2183-C7-2188. 10.1051/jp4:19937348 • jpa-00251996

\section{HAL Id: jpa-00251996 https://hal.science/jpa-00251996}

Submitted on 1 Jan 1993

HAL is a multi-disciplinary open access archive for the deposit and dissemination of scientific research documents, whether they are published or not. The documents may come from teaching and research institutions in France or abroad, or from public or private research centers.
L'archive ouverte pluridisciplinaire HAL, est destinée au dépôt et à la diffusion de documents scientifiques de niveau recherche, publiés ou non, émanant des établissements d'enseignement et de recherche français ou étrangers, des laboratoires publics ou privés. 


\title{
Advanced neutron diffraction techniques for strain measurements in polycrystalline materials
}

\author{
P. MIKULA, P. LUKÁŠ, M. VRÁNA, P. KLIMANEK*, T. KSCHIDOCK* K. MACEK**, \\ J. JANOVEC ${ }^{* *}$, J.C. OSBORN ${ }^{* * *}$ and G.M. SWALLOWE ${ }^{* * *}$
}

Nuclear Physics Institute, 25068 Řež, Czech Republic

${ }^{*}$ Bergakademie Freiberg, 9200 Freiberg, Germany

** Faculty of Mechanical Engineering, CTU, Prague, Czech Republic

*** Physics Dept., Loughborough University of Technology, England

\begin{abstract}
Three unique high resolution experimental arrangements for nondestructive strain measurements which are based on neutron Bragg diffraction optics with cylindrically bent perfect crystals are reviewed. Using focusing in momentum and real space these techniques yield $\Delta d / d$ (d-lattice spacing) resolution of $10^{-4}-10^{-3}$ and considerably higher luminosity in comparison with the current dedicated instruments. They permit measurements not only macrostrain components resulting in angular shifts of diffraction peaks but also of microstrains by means of profilebroadening analysis.
\end{abstract}

\section{INTRODUCTION}

Although focusing principles have been well known in optics for a very long time and have been applied to many research areas, it has taken a relatively long time before focusing techniques with elastically deformed perfect crystals were introduced for neutron scattering instrumentation. However, in the last decade there has been much successful use of them as monochromators [1-4] and in many-crystal systems [.5-11] to obtain a maximum luminosity/resolution ratio for diffraction devices. In this contribution we review the properties of three unconventional nondestructive techniques developed on the basis of Bragg diffraction optics for strain measurements in polycrystalline materials. The techniques may be easily employed on conventional double or triple axis instruments. They use the advantages coming from focusing both in real and momentum space and yield resolution and luminosity comparable with the best powder diffractometers. However, it should be pointed out that such excellent properties of the device may be exploited only in a limited angular range in the vicinity of the chosen Bragg reflection with respect to which all focusing conditions are optimized. Such a situation occurs in the case of strain measurements. The lattice strain is defined as $\varepsilon=\Delta d / d$, where $\Delta d$ is the change in lattice spacing due to strain and $d$ is the unstrained spacing. Differentiation of Bragg's equation, $2 d \sin \theta=\lambda$, where $\theta$ is the diffraction angle, gives $\varepsilon=-\cot \theta . \Delta \theta$ at a fixed wavelength.

\section{THEORETICAL BACKGROUND}

Following the schematic sketch displayed in fig. 1 it is clear that a maximum resolution of this arrangement is achieved for minimum dispersion over the whole system of diffracting elements. When treat- 
ing it in momentum space this means that the orientation of the $\Delta k$ domains (where all the $k$-vectors end) related to the monochromator and analyzer are matched to that of the sample.

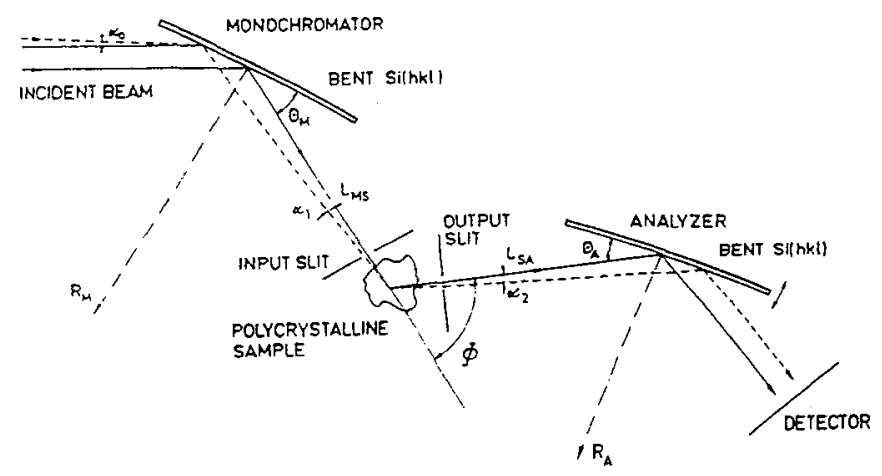

Fig.1. Schematic sketch of the triple axis setup with bent perfect monochromator and analyzer.

The condition of focusing in momentum space for the whole setup can be written in a simple form [10]

$$
2 \tan \theta_{S}=\frac{\tan \theta_{M}}{1-L_{M S} / \overline{2} \bar{f}_{M}}+\frac{\tan \theta_{A}}{I-L_{S A} / \overline{2} \bar{f}_{A}},
$$

where $L_{\mathrm{MS}} L_{\mathrm{SA}}$ are the monochromator-sample, sample-analyzer distances and $f_{M^{\prime}} f_{A}$ are the focal lengths of the monochromator and the analyzer $\left(f_{M(A)}=R_{M(A)} \sin \theta_{M(A)} / 2\right)$. The mutual relations between the angles $\alpha_{o}$, $\alpha_{1}$ and $\alpha_{2}$ are given by

$$
\begin{gathered}
\alpha_{1}=2 \varepsilon\left(R_{M}\right)-\alpha_{0}, \\
\alpha_{2}=\alpha_{1}\left[2 \frac{\tan \theta_{S}}{\tan \theta_{M}}\left(1-L_{M S} / 2 f_{M}\right)-1\right],
\end{gathered}
$$

where $R_{M}$ is the monochromator bending radius and $\varepsilon\left(R_{M}\right)=L_{M S} \alpha_{1} / 2 f_{M}$ is the bending angle over the whole length of the irradiated crystal slab. Inspection of equations (1)-(3) reveals that for standard experimental conditions with a soller collimator in the incident polychromatic beam the maximum luminosity of the instrument is achieved when $L_{\mathrm{HS}}=f_{\mathrm{M}}$ and $\alpha_{\mathrm{O}}=0$.

\section{EXPERIMENTAL TECHNIQUES}

For the experiment we use si perfect single crystal slabs of dimensions $5 \times 30 \times 200 \mathrm{~mm}^{3}$ (thickness $x$ height $x$ length) with the main surface parallel to the 110,111 or 112 lattice planes. The slabs are bent by a four-point bending device, enabling a reproducible adjustment of radii ranging from $9 \mathrm{~m}$ to infinity (flat crystal). The dedicated triple axis spectrometer has fixed distances $L_{M S}=135 \mathrm{~cm}$ and $L_{S A}=56 \mathrm{~cm}$. A rather short distance $L_{\mathrm{SA}}$ brings some limitations in optimization of the instrument according to (1). Sampled volume was determined by two $2 \mathrm{~mm}$ wide Cd slits placed near to the sample in the incident as well as diffracted beams. 


\subsection{High resolution three axis arrangement}

This arrangement corresponds to the one sketched in fig. 1, where both crystals are in symmetric Bragg reflection geometry. In order to fulfil the condition (1) we tested three combinations of reflections of the monochromator and the analyzer with respect to a chosen reflection of an $\alpha$-iron sample at $\lambda=0.162 \mathrm{~nm}$ (see fig. 2). The measurement consists of performing the $\phi$-scan $\left(\phi=2 \theta_{S}\right)$ keeping the $\theta_{A}$-angle fixed. Using the nearly nondispersive combination $\mathrm{Si}(220) / \mathrm{Fe}(110) / \mathrm{Si}(220)$ when $d_{220}^{\text {Si }}$ and $d_{110}^{F e}$ differ only by 5.4 $\%$, eq. (1) is fulfilled with a good accuracy in a large range of $R$, where simultaneously $L_{\mathrm{MS}} / 2 f_{\mathrm{M}} \ll 1, L_{\mathrm{SA}} / 2 f_{\mathrm{A}} \ll 1$. Even though this combination does not exploit focusing in real space, it gives the maximum resolution for the Fe Bragg peak ( $F W H M=6.1^{\prime}$ ) and Yields sufficient intensity usable for practical measurements $[9,10]$.

Almost the same $F W H M=7.8^{\prime}$ as in the former case, but with a considerably higher intensity (by a factor of 6) was achieved with the combination $\mathrm{Si}(111) / \mathrm{Fe}(110) / \mathrm{Si}(220)$. In this case $L_{M S} \simeq f_{M}$ and $\tan \theta_{s} \simeq 2 \tan \theta_{M}$. Owing to the

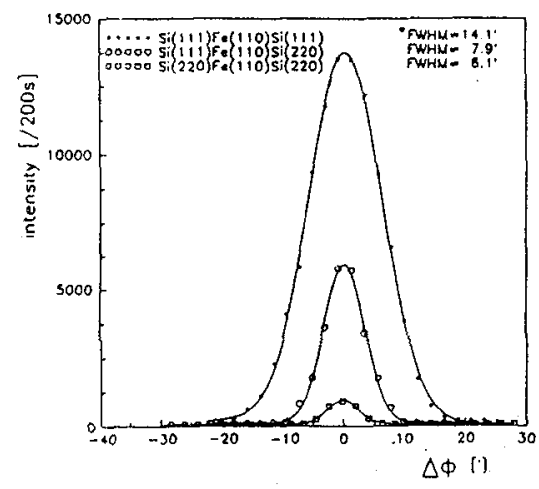

Fig.2. Intensity and resolution comparison of different combinations of monochromators and analyzers $10.1 \mathrm{~cm}^{3}$ volume of the sample). lower Bragg angle at the monochromator, neutrons from a larger irradiated volume are delivered onto the sample. Since in our case due to a rather small distance of $L_{\mathrm{SA}}=56 \mathrm{~cm}$, $L_{S A} \ll f_{A}$ is still valid, the eq. (1) is fulfilled when using $S i(220)$ analyzer. Due to the high resolution of both the above combinations they may be succesfully employed for plastic strain studies using shape analysis of the diffraction profile [12].

However, the combination $\mathrm{Si}(111) / \mathrm{Fe}(110) / \mathrm{Si}(111)$ appears most $1 \mathrm{u}-$ minous, because it exploits simultaneously focusing in real and momentum space for both monochromator and analyzer. The gain in peak intensity as compared to the first combination is about a factor of 14 even though the resolution is redused by a factor of 2.3 (FWHM=14.1').

Fig. 3 and 4 demonstrate examples of the practical employment of the second modification.

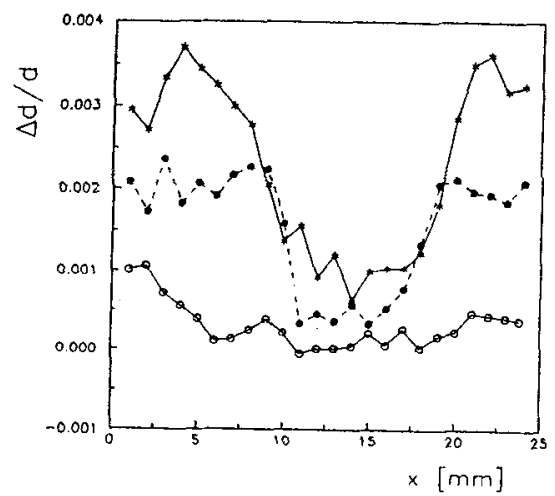

Fig.3. Scan of residual stress through the weld joln in a high alloy steel sample before (*) and after annealing at $520^{\circ} \mathrm{C}(0)$ or $650^{\circ} \mathrm{C}(0)$.



Fig.4. Diffraction peaks of a stretched Cu-sample for two values of loading and Si(111)/Cu(111)/Si(220) arrangement. 
3.2 Three axis arrangement with the asymmetric analyzer in combination with PSD

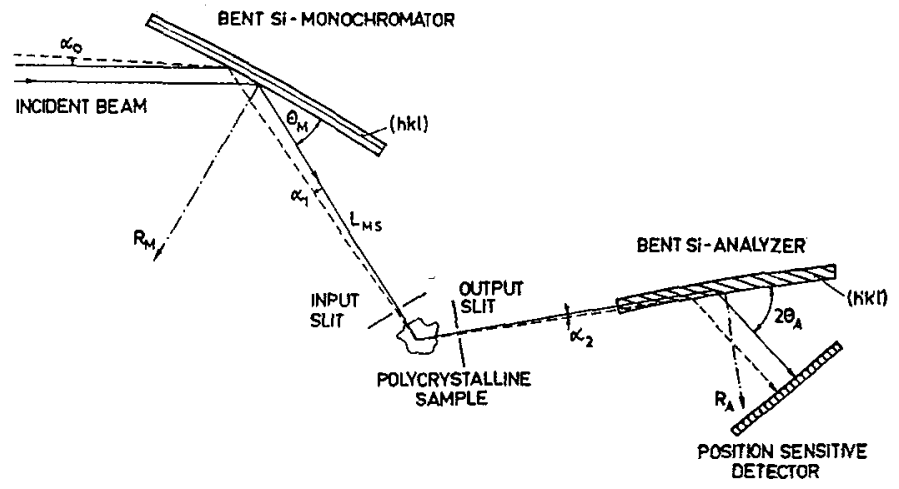

Fig.5. Schematic sketch of the arrangement with the asymmetric analyzer In combination with PSD.

This arrangement, schematically sketched in fig 5 , uses fully asymmetric diffraction (FAD) geometry of the bent-crystal analyzer already used for medium resolution sANs measurements $[6,8]$. Owing to this unconventional geometry the Bragg angle varies homogeneously along its longest edge. In this way the deviation from the mean Bragg angle is transformed onto the linear spatial scale which in fact enables us to measure conventional diffraction profiles in "one step" by means of a 1d-PSD. Using a crystal slab we had at our disposal, we tested two reflection combinations: $S i(111) / \mathrm{Fe}(110) / \mathrm{Si}(11 \overline{1})$ for $\lambda=0.21$ $\mathrm{nm}$ and $\mathrm{Si}(111) / \mathrm{Fe}(110) / \mathrm{Si}(022) \quad \lambda=0.22 \mathrm{~nm}$ (see fig. 6). Fig. 7 demonstrates simulation of the macrostrain by heating the $\alpha$-iron sample to a temperature of about $386 \mathrm{~K}$. It can be seen from fig. 7 that using the PSD the reflection profile (with a sufficient numbers of counts necessary for determination of $\Delta d / d$ with an accuracy of about $10^{-4}$ ) for $0.1 \mathrm{~cm}^{3}$ sampled volume is measured with a medium power reactor in several minutes.

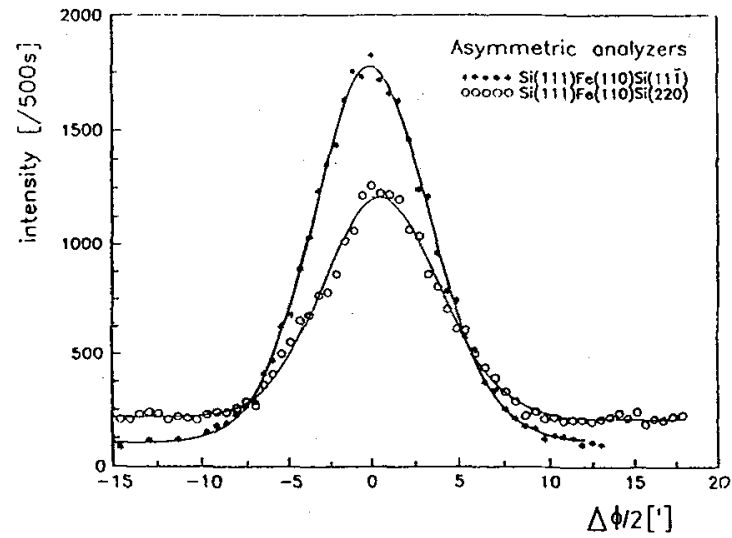

Fig.6. Comparison of diffraction profiles obtained with two different analyzers in FAD geometry.

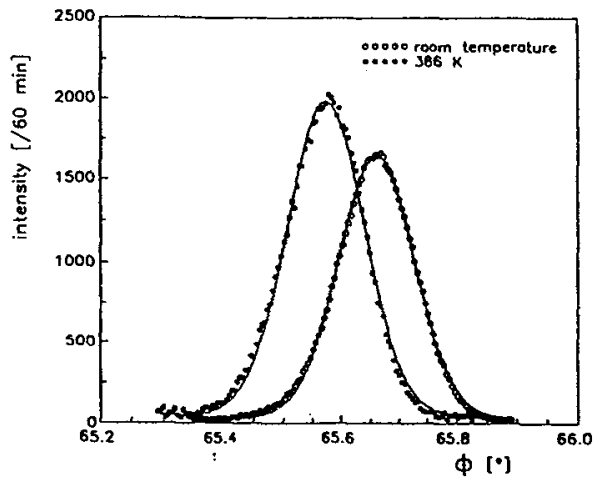

Fig.7. Reflection profile of a polycrystalline Fe(110) analyzed by the analyzer in FAD geometry employed in the combination $\mathrm{Si}(111) / \mathrm{Fe}(110) / \mathrm{Si}(11 \overline{1})$.

\subsection{Two axis arrangement with the "parallel" diffracted beam}

This unique arrangement (see fig. 8) exploits excellent focusing properties of cylindrically bent perfect crystals in real and momentum space with respect to samples of small width. 


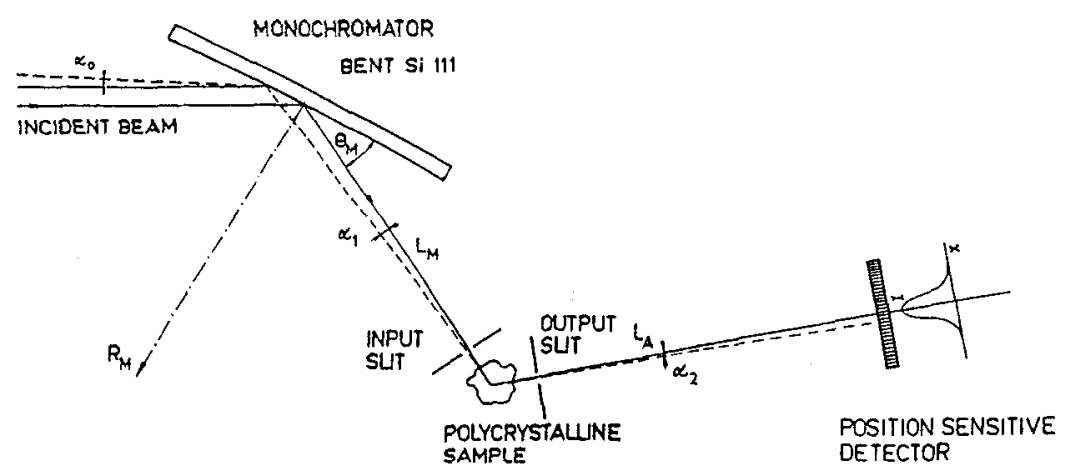

Fig.8. Schematic sketch of the "paralle1" beam arrangement.

Setting $\alpha_{2}=0$ in (3) we arrive at the condition for $R_{M}$ as

$$
R_{M}=\frac{2 L_{M S}}{\sin \theta_{M}} \frac{\tan \theta_{S}}{2 \tan \theta_{S}-\tan \theta_{M}} .
$$

However, owing to the nonnegligible thickness of the bent monochromator as well as the width of the sample, the uncertainties introduced [12] cause the diffracted beam from the sample to be slightly. divergent (see fig. 9). Nevertheless, the FWHM of the diffraction profile is comparable to or smaller than that of conventional dedicated instruments. The high luminosity of such an instrument (the absence of soller collimators, the use of a high resolution PSD) predicts this two axis arrangement for fast acquisition of experimental data, particularly from elastic strain measurements. Fig. 10 demonstrates the effectiveness of this arrangement on the $\alpha$-iron etalon and compressed martensite samples.

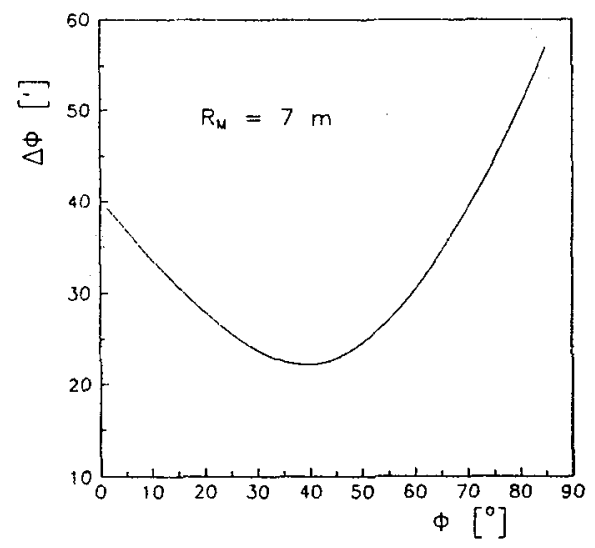

Fig.9. Theoretical dependence of the uncertainty $\Delta \Phi$ on $\dot{\Phi}$ for $\lambda=0.162 \mathrm{~nm}, \quad R=7 \mathrm{~m}$ and monochromator thlckness of $3 \mathrm{~mm}[13]$.

\section{CONCLUSION}

Several experimental arrangements suitable for employment in high resolution powder diffractometry are introduced. Using the optical properties of bent perfect crystals one may obtain unconventionally high angular resolution with high luminosity. Owing to the necessity of tuning the whole arrangement with respect to particular reflection 
of a sample of necessarilly small width, the main applicability of the presented arrangements is for the high resolution investigations in a rather small Q-range or in the vicinity of individual reflections. For more details see ref. 13 .

\section{References}

[1] Mikula P., Kulda J., Vrána M. and Chalupa B., J. Appl. Cryst. 17 (1984) 189-195.

[2] Mikula P., Lukáš P. and Michalec R., J. Appl. Cryst. 20 (1987) 428-430.

[3] Mikula P., Krüger E., Scherm R. and Wagner V., J. Appl. Cryst. 23 (1990) 105-110.

[4] Wagner V. and Mikula P., In Proc. of SPIE's 1992 International Symp. on Optical Applied Science and Engineering, San Diego, 22-24 July, 1738 pp. 431-437.

[5] Kulda J. and Mikula P., J. Appl. Cryst. 16 (1983) 498-504.

[6] Mikula P., Lukáš P. and Eichhorn F., J. Appl. Cryst. 21 (1988) 33-37.

[7] Mikula P., Wagner V. and Scherm R., J. Appl. Cryst. 24 (1991) 298-303.

[8] Mikula P., Lukáš P., Kulda J., Strunz P. and Saroun J., In Proc. of SPIE's 1992 International Symp. on optical Applied Science and Engineering, San Diego, 22-24 July 1992, 1738 pp. 411-421.

[9] Kulda J., Mikula P., Lukáš P. and Kocsis M., Physica B 180 \& 181 (1992) 1041-1043.

[10] Lukáš P., Vrána M., Mikula P. and Kulda J., In Proc. of SPIE's 1992 International Symp. on Optical Applied Science and Engineering, San Diego, 22-24 July 1992, 1738 pp. 438-443.

[11] Popovici M. and Yelon W.B., In Proc. of SPIE's 1992 International Symp. on optical Applied Science and Engineering, San Diego, 22-24 July, $1738 \quad 422-430$.

[12] Klimanek P., Kschidock T., Lukáš P., Mikula P., Mücklich A. and Vrána M., In Proc. 3rd European Conference on Advanced Materials and Processes - EUROMAT 93, Paris (1993), within this issue.

[13] Wagner V., Mikula P. and Lukáš, P., In Proc. of the 105th PTB Seminar and ILL Workshop "Focusing Bragg optics", PTB Braunschweig 10-11 May, 1993; submitted to Nucl. Instrum. Methods. 